\title{
Study of the human hypoglossal nucleus: Normal development and morpho-functional alterations in sudden unexplained late fetal and infant death
}

\author{
Anna Maria Lavezzi *, Melissa Corna, Rosaria Mingrone, Luigi Matturri \\ "Lino Rossi" Research Center for the Study and Prevention of Unexpected Perinatal Death and SIDS - Department of Surgical, \\ Reconstructive and Diagnostic Sciences, University of Milan, Via della Commenda, 19, 20122 Milan, Italy
}

Received 23 February 2009; received in revised form 20 May 2009; accepted 25 May 2009

\begin{abstract}
This study evaluated the development and the involvement in sudden perinatal and infant death of the medullary hypoglossal nucleus, a nucleus that, besides to coordinate swallowing, chewing and vocalization, takes part in inspiration. Through histological, morphometrical and immunohistochemical methods in 65 cases of perinatal and infant victims (29 stillbirths, 7 newborns and 29 infants), who died of both unknown and known cause, the authors observed developmental anomalies of the hypoglossal nucleus (HGN) in high percentage of sudden unexplained fetal and infant deaths. In particular, HGN hypoplasia, hyperplasia, positive expression of somatostatin and absence of interneurons were frequently found particularly in infant deaths, with a significant correlation with maternal smoking.
\end{abstract}

(C) 2009 Elsevier B.V. All rights reserved.

Keywords: Cigarette smoking; Human hypoglossal nucleus; Neuropathology; SIDS; Unexplained fetal death

\section{Introduction}

Developmental abnormalities of brainstem centers checking the vital functions have been reported by us in sudden perinatal and infant death victims. These include, in particular in the brainstem, hypoplasia of the arcuate nucleus, pre-Bötzinger nucleus, tractus solitarius nucleus, parafacial complex and parabrachial/ Kölliker-Fuse complex [1-7]. The present study was performed with the purpose to deepen our knowledges by evaluating the development and the involvement in these pathologies even of the hypoglossal nucleus (HGN), a nucleus of the dorsal part of the medulla oblongata that, even if not generally included among the so defined vital centers, takes part in specific phases of breathing. In fact

\footnotetext{
* Corresponding author. Tel.: +39 02 50320821; fax: +39 02 50320823

E-mail address: anna.lavezzi@unimi.it (A.M. Lavezzi).
}

the hypoglossal neurons, besides to coordinate swallowing, chewing and vocalization, have been identified as having a peak of activity during inspiration [8-10].

The HGN contains two distinct neuronal populations: the first, predominant, is represented by motoneurons, that are large, multipolar and rich in Nissl substance neurons; the second neuronal pool is constituted by small round or oval neurons with poor rough endoplasmatic reticulum, defined as "inhibitory interneurons" [11-13].

The functional significance of the two neuronal groups in HGN has been explained in relation to tongue control, in particular to control of the genioglossus muscle that is important in maintaining a patent airway, especially during inspiration [14-16]. Both the HGN motoneurons and interneurons are involved in the generation of excitatory and inhibitory post-synaptic potential during the different above-mentioned functions that require precise coordination of tongue movements. 
The current knowledges on the HGN are prevalently obtained from experimental studies. A review of the literature shows that only few authors, and many years ago, have studied the HGN in man, precisely in sudden infant death syndrome (SIDS) [17-20]. They observed significantly higher incidence of morphopathological features of this nucleus in victims of sudden infant death than in control infants.

Therefore, we aimed to start from these works to evaluate if the HGN shows morpho-functional alterations not only in SIDS but also in sudden unexplained perinatal deaths, like the alterations in nuclei and/or structures of the brainstem and cerebellum checking vital functions, previously observed by us in these pathologies [1-7,21-23].

Thus, we firstly investigated the developmental patterns of the HGN in a wide cohort of fetal and infant death victims aged from the 17th gestational week to 10 months of life, who had died of known and unknown causes, and then we evaluated whether morphofunctional disorders could be present in this nucleus in victims of unexplained death.

The study protocol of the HGN included, in all cases: (1) morphological examination in histological sections of medulla oblongata; (2) morphometrical analysis of nucleus area and neuronal density; (3) immunohistochemical evaluation of the expression of the somatostatin, a neurotransmitter that controls important physiological functions already from the first weeks of gestation.

Finally, the observation in our previous studies of a significantly increased incidence of structural and/or functional alterations of the central autonomic nervous system in victims of unexplained perinatal and infant death with smoker mothers [21,24-26], prompted us to determine whether prenatal absorption of nicotine could also interfere with the maturational processes of the HGN.

\section{Patients and methods}

A total of 65 brains were collected from 29 fresh antepartum stillbirths (17-40 gestational weeks, with a peak from 36 to 40 weeks), 7 newborns who died within the first two days of life and 29 infants aged 1-10 months (mean age: 3.5 months).

For every case, a complete clinical history, particularly referred to the mothers, and including the death scene examination in infant victims, was collected.

None of the mothers had any significant pathology. While taking the medical history, the mothers were asked for information about any smoking habit before, during and after pregnancy. Characteristics of the mothers per smoking category were: $11 \%(n=7)$ reported smoking only during pregnancy, $23 \%(n=15)$ smoking before, during and after pregnancy and 55\% $(n=36)$ no smoking. In 7 cases $(11 \%)$ no information about smoking was available.

The victims were subjected, with a mean post-mortem interval of $24 \mathrm{~h}$, to a complete autopsy, including examination of the placental disk, umbilical cord and membranes in perinatal deaths. In all cases an in-depth histological examination of the autonomic nervous system was made, according to the protocol routinely followed by the "Lino Rossi Research Center for the study and prevention of the unexpected perinatal death and SIDS" of the Milan University [27,28].

In particular, after fixation in $10 \%$ phosphate-buffered formalin, the brainstem, where the main structures checking vital functions are located, was processed and embedded in paraffin. Transverse serial sections of the midbrain, pons and medulla oblongata were made at intervals of $60 \mu \mathrm{m}$. For each level, five $5 \mu \mathrm{m}$ sections were obtained, three of which were stained for histological and morphometrical examination using hematoxylin-eosin, Klüver-Barrera stains and silver impregnation technique of Bielchowsky. Two additional sections were subjected to immunohistochemistry for the study of the somatostatin.

The in-depth examination of the hypoglossus nucleus, the target of this study, was performed in all the cases at the same level of the medulla oblongata, and precisely on the histological sections corresponding to the obex [28]. This section also allow to easily analyze further important medullary structures (dorsal motor vagus, tractus solitarius, ambiguous, pre-Bötzinger, inferior olivary and arcuate nuclei). In the pons we analyzed the locus coeruleus, the parabrachial/KöllikerFuse complex, the facial/parafacial complex and the superior olivary complex.

In 35 cases, after the in-depth autoptic examination, the death remained totally unexplained. A diagnosis of "unexplained stillbirth" was established for 16 fetuses, who died suddenly after the 17th gestational week before complete expulsion or retraction from the mother; a diagnosis of "unexplained early neonatal death" for 4 newborns who died between birth and the first two postnatal days, and of SIDS for 15 infants died within the first ten months of life. In the remaining 30 cases, 13 stillbirths, 3 newborns and 14 infants, a precise cause of death was formulated at autopsy. Therefore, these cases were regarded as "controls".

Table 1 summarizes the case profiles in this study, indicating the sex distribution, range of ages and death diagnoses.

\subsection{Morphometric analysis}

The morphometric analysis was performed with an Image-ProPlus Analyser (Media Cybernetics, Silver Spring, MD). The histological image acquisition was made via a CCD Color Camera (Ikegami Tsushinki 
Table 1

Case profiles in the study.

\begin{tabular}{|c|c|c|c|c|c|}
\hline \multirow[t]{2}{*}{ Victims } & \multirow[t]{2}{*}{ Age (range) } & \multicolumn{2}{|c|}{$\operatorname{Sex}(n)}$. & \multicolumn{2}{|l|}{ Death diagnosis } \\
\hline & & M & $\mathrm{F}$ & Explained deaths (n.30) & Unexplained deaths (n.35) \\
\hline Fetuses (n.29) & $17-40 \mathrm{gw}$ & 12 & 15 & $\begin{array}{l}\text { Necrotizing chorioamnionitis (n.7) } \\
\text { Congenital heart diseases (n.5) } \\
\text { Potter's syndrome (n.1) }\end{array}$ & Unexplained stillbirth (n.16) \\
\hline Newborns (n.7) & $1-4 \mathrm{pd}$ & 4 & 3 & Congenital heart diseases (n.3) & Unexplained early neonatal death (n.4) \\
\hline Infants (n.29) & $1-10 \mathrm{~m}$ & 16 & 13 & $\begin{array}{l}\text { Pneumonia (n.6) } \\
\text { Congenital heart diseases (n.7) } \\
\text { Pericarditis (n.1) }\end{array}$ & SIDS (n.15) \\
\hline
\end{tabular}

$\mathrm{gw}=$ Gestational week; $\mathrm{pd}=$ postnatal day; $\mathrm{m}=$ month.

SIDS $=$ Sudden infant death syndrome.

Co., Ltd, Ohta-ku, Tokyo, Japan), using a Plan Neofluar Zeiss objective and were displayed in a PC-monitor in $\mathrm{RGB}$ real colour.

The measurements were made in a blinded fashion, without knowledge of the clinical diagnosis or victim age, in all cases in the same histological sections, selected at the anatomic level of the obex. The following parameters were evaluated:

- Surface area: Surface area of the HGN $\left(\mathrm{mm}^{2}\right)$. The outer boundary of the nucleus was delineated on both side of medulla oblongata.

- Neuronal density: Neuronal density of the HGN (number of cells $/ \mathrm{mm}^{2}$ ). The neurons were counted on both sides of the sections using a $\times 40$ objective. The number of cells was then correlated with the HGN area, obtaining the cells density.

The measured parameters were expressed as mean and standard deviation (SD).

\subsection{Somatostatin immunohistochemistry}

Lyophilized rabbit serum diluted in PBS (Novocastlab-Newcastle, UK) was used in this study. This antiserum recognized the N-terminal part of SS-28, composed of 28 aminoacids. Somatostatin immunoreactivity was visualized by the peroxidase-antiperoxidase method. In order to neutralize endogenous peroxidase, sections were pretreated with a solution of $0.3 \%$ hydrogenase peroxide for $20 \mathrm{~min}$. After rinsing in buffer, sections were exposed for $48 \mathrm{~h}$ to the specific primary antiserum diluted $1: 150$ at $25^{\circ} \mathrm{C}$. After $10 \mathrm{~min}$ in buffer, the site of antigen-antibody reaction was revealed with antirabbits immunoglobuline followed by peroxidase-antiperoxidase complex. The unlabeled antiserum was placed on the sections for $2 \mathrm{~h}$ diluted 1:200 at room temperature. The sections were then exposed to peroxidase-antiperoxidase complex for $2 \mathrm{~h}$ at dilution 1:200. The sites of peroxidase activity were visualized with $0.3 \%$ hydrogen peroxide in buffer containing $0.04 \%$ diaminobenzidine tetrahydrochloride and $0.5 \mathrm{~g}$ nickel ammonium sulfate. Slides were rinsed, dehydrated, mounted and examined by light microscopy.

\subsection{Statistical analysis}

The statistical significance of direct comparison between the groups of victims was determined using analysis of variance (ANOVA). Statistical calculations were carried out on a personal computer with SPSS statistical software. The selected threshold level for statistical significance was $p<0.05$.

\section{Results}

\subsection{Morpho-functional steps of the human $H G N$ development}

\subsubsection{Morphological features}

At the earliest observation (17-18 gestational weeksgw) the HGN was scarcely delineated and consisted of few round neurons with large nucleus, evident nucleolus and poor cytoplasm (Fig. 1a,b). Several mitoses were detected at this developmental stage.

From the 20th to the 22nd gw, the HGN showed a dense group of small cells, with eccentric nucleus and granular cytoplasm. Several polygonal neurons with evident axons and dendrites were observed (Fig. 2a,b).

At $30 \mathrm{gw}$ the HGN was well recognizable, and consisted of intermingled polygonal and round neurons spread in a wide area. The multipolar neurons showed dense lamellar arrays of rough endoplasmic reticulum (Nissl substance) in the cytoplasm (Fig. 3). These features were emphasized around the 35 th gw, with increase in neuronal number and neuropil expansion (Fig. 4).

After birth, we observed two different and well defined morphologies of HGN neurons. The predominant population was constituted by large multipolar neurons, with round eccentric nucleus and cytoplasm rich in Nissl substance. The second population consisted of small round to oval shaped neurons with frequently 

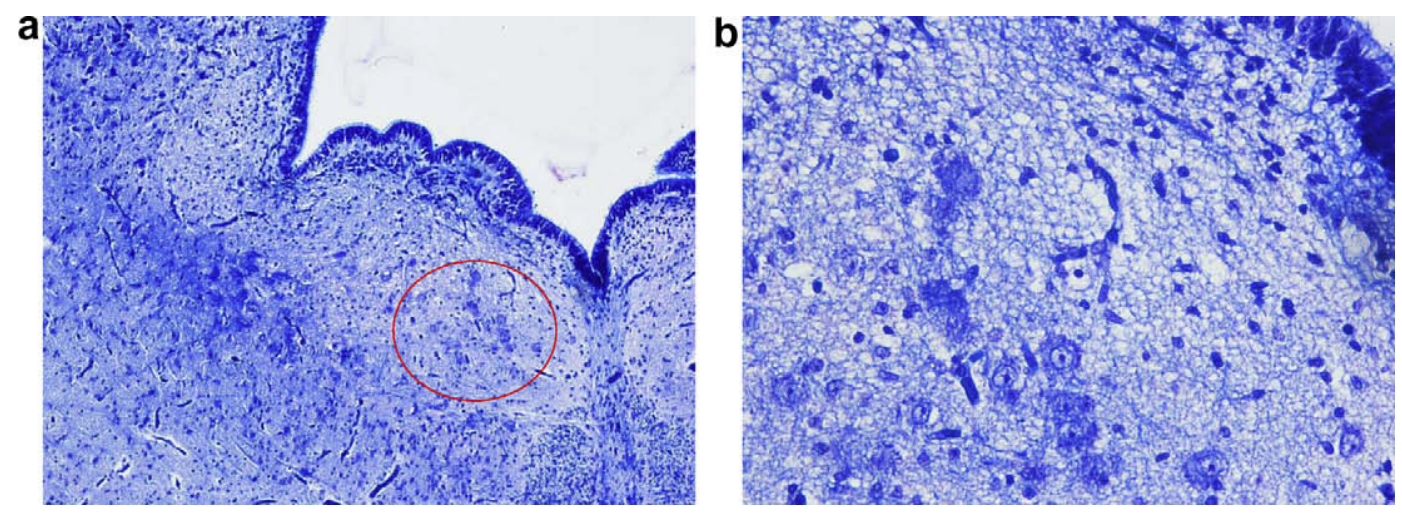

Fig. 1. Hypoglossal nucleus in a fetus of the control group aged 17 gestational weeks. The nucleus is scarcely delineated and consists, as better observable in (b), of few round neurons with large nucleus, evident nucleolus and poor cytoplasm. Klüver-Barrera stain. Magnification: (a) 4×; (b) $20 \times$.
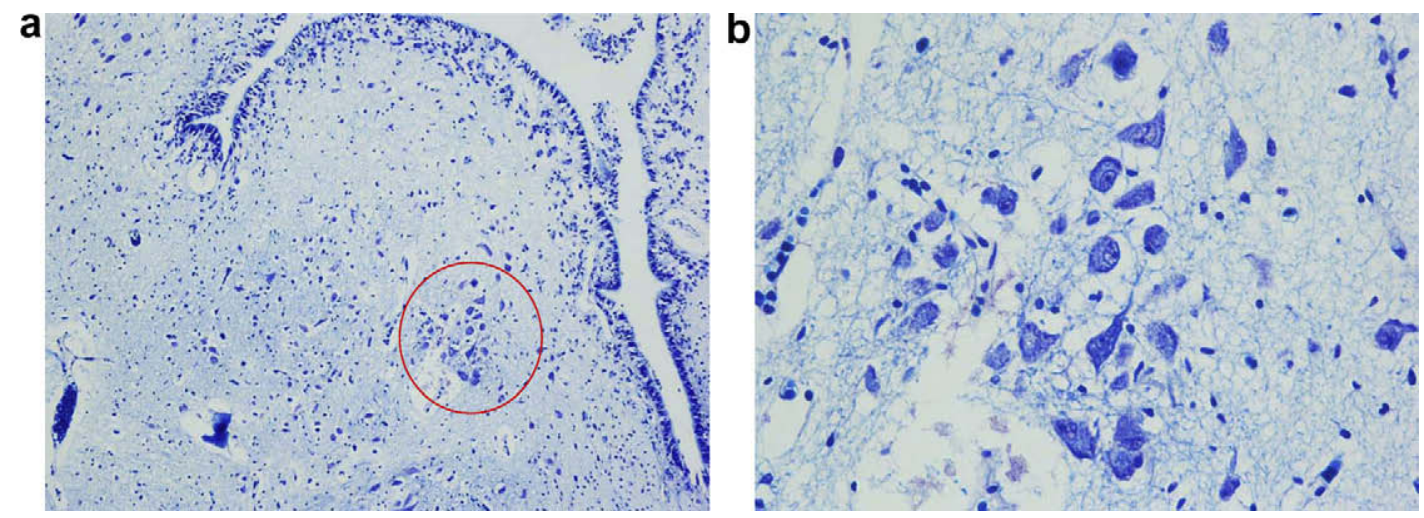

Fig. 2. Hypoglossal nucleus in a fetus of the control group aged 22 gestational weeks. The neurons, as better observable in (b), shows eccentric nucleus and granular cytoplasm. Several neurons are polygonal in shape, with evident axons and dendrites. Klüver-Barrera stain. Magnification: (a) $4 \times$; (b) $20 \times$.

eccentric nucleus, scant cytoplasm, prevalently assembled in the ventral region of HGN (Fig. 5a,b). These two types of neurons were classified as "motoneurons" and "interneurons", respectively.

\subsubsection{Morphometric features}

Table 2 shows the HGN areas and neuronal density obtained through morphometric analysis. Because of the low number of neonatal deaths and the superimposable morphometric values obtained from victims died ante-partum and in the first days post-partum, we subdivided the victims of the study into four groups: I-unexplained perinatal (fetal + neonatal) deaths; II-control perinatal deaths; III-SIDS; IV-control infant death. No statistically significant difference was found between the morphometric parameters of the groups I and II, and between the groups III and IV $(p>0.05)$. On the contrary, there was a marked increase of the HGN outlines and a decrease of the neuronal density during development from the perinatal groups (I and II) to infant groups (III and IV) $(p<0.05)$.

\subsubsection{Somatostatin expression features}

The immunohistochemical study of the somatostatin showed a peculiar positivity for this neuropeptide in the brainstems. In fact, in stillbirths an intense expression was constantly seen in the cell bodies and fibers of the major part of the nuclei, including hypoglossal nucleus.

In the postnatal deaths we observed an abrupt reduction of somatostatin-positivity. In fact, even in the first days of life, we observed a low number of positive cell bodies limited to several nuclei, such as the tractus solitarius nucleus in the medulla oblongata and the locus coeruleus in the pons.

We found the above-described sequence of HGN developmental steps in the large part of cases, particularly in all the control cases. Nevertheless, in 24 victims of unexplained death $(69 \%)$, aged from $36 \mathrm{gw}$ to 4 months of life (10 late fetal deaths, and 14 infant deaths), we observed different structural and biological patterns as compared with agematched subjects. 


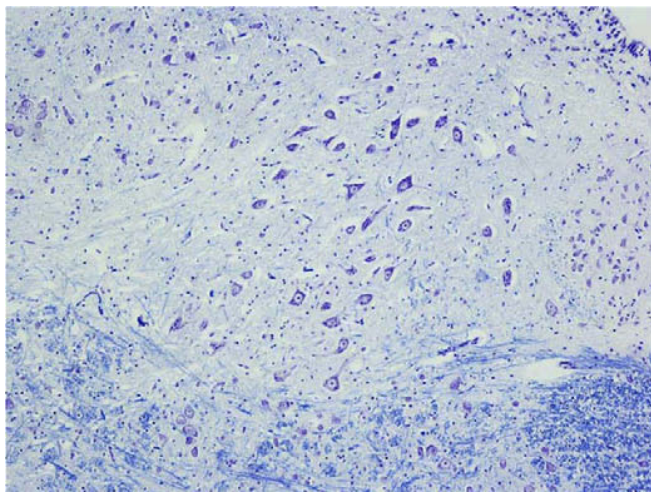

Fig. 3. Hypoglossal nucleus in a fetus of the control group aged 30 gestational weeks. Klüver-Barrera stain. Magnification: 10×.

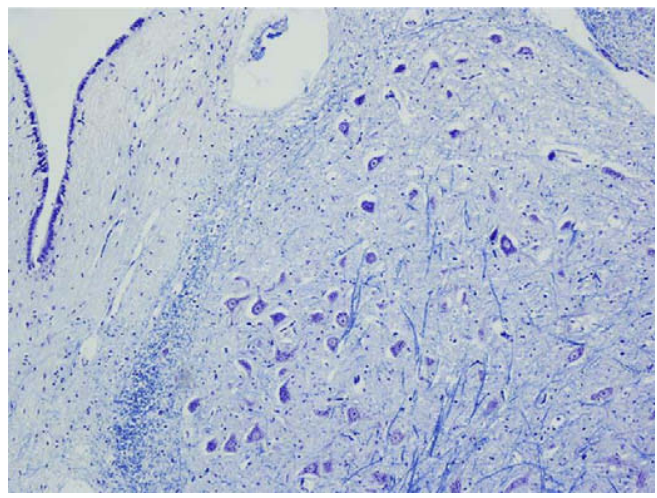

Fig. 4. Hypoglossal nucleus in a fetus of the control group aged 35 gestational weeks. Klüver-Barrera stain. Magnification: $10 \times$.

\subsection{Neuropathology of the $H G N$ in unexplained fetal death and SIDS}

The HGN of 10 fetuses who died in the last weeks of pregnancy, showed the same immature structure that may be found at 22-23 gestational weeks, with increased neuronal density (from 82 to 95 neurons $/ \mathrm{mm}^{2}$ ) due to scanty neuropil. The neurons were in prevalence roundish in shape, with either smooth or rough cytoplasm and sketchy axons. The immunohistochemical evaluation of the somatostatin was negative in 6 of these cases.

In 14 SIDS cases we found different pathological patterns of the HGN, and precisely, as shown in Table 3:

- Hyperplasia: with very large HGN area due to increased number of both motoneurons and neuropil surface, observed in 5 cases (Fig. 6a,b);

- hypoplasia: with small HGN area, decreased motoneuronal number and neuropil, observed in 4 cases (Fig. 7a,b);

- lack of interneurons: with normal or increased HGN boundaries as regards to standard values, and the only presence of large multipolar neurons, observed in 8 cases;

- somatostatin immunopositivity: with marked expression of this neurotransmitter in the HGN neurons, that instead should be immunonegative, in 6 cases (Fig. 8), three of these showing also HGN hyperplasia without interneurons, and one HGN hypoplasia.

Only two victims of the infant control group, died of pneumonia, showed HGN hypoplasia. On the whole, the incidence of the observed HGN alterations was significantly higher in SIDS victims than in infant controls $(p<0.01)$.

In 8 of the SIDS victims with developmental abnormalities of the HGN, we also observed alterations of different brainstem and cerebellum structures (hypoplasia/ agenesis of the arcuate nucleus and of the pre-Bötzinger nucleus, cerebellar cortex delayed maturation and dentate nucleus hyperplasia). The most frequent association was with the hypodevelopment of the medullary arcuate nucleus.

With regard to fetal death victims, 8 of the 10 cases with delayed development of the HGN showed hypoplasia of the pontine facial/parafacial complex.
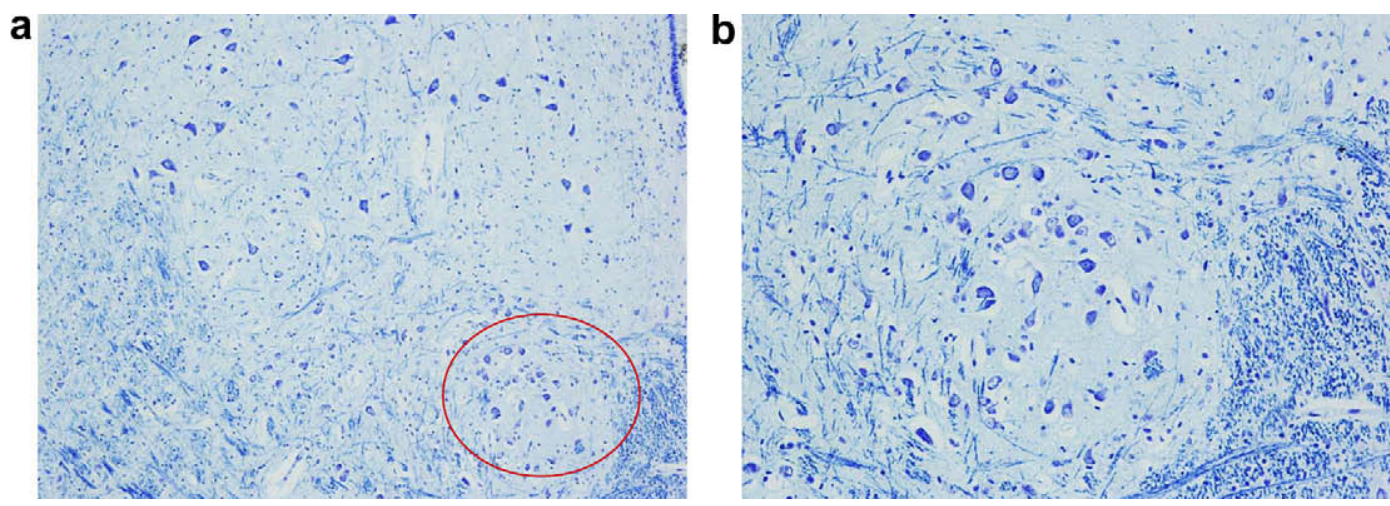

Fig. 5. Hypoglossal nucleus in an infant of the control group aged 1 month. In the encircled area in (a): a group of interneurons. They appear at greater magnification in (b). Klüver-Barrera stain. Magnification: (a) 4×; (b) $20 \times$. 
Table 2

Morphometric analysis of the HGN in perinatal and infant deaths.

\begin{tabular}{|c|c|c|c|c|}
\hline \multirow[t]{2}{*}{ Morphometric parameter } & \multicolumn{4}{|l|}{ Victim groups } \\
\hline & $\begin{array}{l}\text { I Unexplained perinatal } \\
\text { death }(\mathrm{n} .20)\end{array}$ & $\begin{array}{l}\text { II Control } \\
\text { perinatal death (n.16) }\end{array}$ & $\begin{array}{l}\text { III SIDS } \\
(\mathrm{n} .15)\end{array}$ & $\begin{array}{l}\text { IV Control infant } \\
\text { death (n.14) }\end{array}$ \\
\hline HGN area $\left(\mathrm{mm}^{2}\right)$ mean $\pm \mathrm{SD}$ & $0.444 \pm 0.11$ & $0.477 \pm 0.16$ & $0.851 \pm 0.44$ & $0.775 \pm 0.19$ \\
\hline $\begin{array}{l}\text { HGN neuronal density } \\
\left(\text { neuron number } / \mathrm{mm}^{2} \text { ) mean } \pm \mathrm{SD}\right.\end{array}$ & $74 \pm 2$ & $72 \pm 9$ & $51 \pm 11$ & $49 \pm 3$ \\
\hline
\end{tabular}

HGN = hypoglossal nucleus; $\mathrm{SD}=$ standard deviation.

Significance of group I vs group II and of the group III vs group IV: $p>0.05$.

Significance of perinatal deaths (groups I and II) vs infant deaths (groups III and IV): $p<0.05$.

Table 3

HGN pathological findings in 14 victims of SIDS.

\begin{tabular}{|c|c|c|c|c|c|c|}
\hline \multirow[t]{2}{*}{ Case $n$. } & \multirow[t]{2}{*}{ Sex/age (months) } & \multicolumn{4}{|c|}{ HGN alteration } & \multirow{2}{*}{$\begin{array}{l}\text { Further neuropathological } \\
\text { findings }\end{array}$} \\
\hline & & Hyperplasia & Hypoplasia & $\begin{array}{l}\text { Lack of } \\
\text { interneurons }\end{array}$ & $\begin{array}{l}\text { Somatostatin } \\
\text { immunopositivity }\end{array}$ & \\
\hline 1 & $\mathrm{M} / 1$ & + & - & + & + & $\begin{array}{l}\text { ARCN agenesis } \\
\text { pBN hypoplasia }\end{array}$ \\
\hline 2 & $\mathrm{~F} / 1$ & - & + & - & + & $\mathrm{pBN}$ agenesis \\
\hline 3 & $\mathrm{M} / 2$ & - & - & + & - & - \\
\hline 4 & $\mathrm{M} / 2$ & + & - & - & + & ARCN hypoplasia \\
\hline 5 & $\mathrm{M} / 2$ & + & - & - & + & - \\
\hline 6 & $\mathrm{~F} / 3$ & - & - & + & - & ARCN hypoplasia \\
\hline 7 & $F / 3$ & - & - & + & - & - \\
\hline 8 & $\mathrm{M} / 3$ & - & - & + & - & - \\
\hline 9 & $\mathrm{M} / 3$ & - & + & - & - & ARCN hypoplasia \\
\hline 10 & $\mathrm{~F} / 3$ & - & - & + & - & $\begin{array}{l}\text { ARCN hypoplasia } \\
\text { CC immaturity }\end{array}$ \\
\hline 11 & $\mathrm{M} / 3$ & + & - & + & + & - \\
\hline 12 & $\mathrm{~F} / 4$ & - & + & - & - & ARCN hypoplasia \\
\hline 13 & $\mathrm{M} / 4$ & - & + & - & - & - \\
\hline 14 & $\mathrm{M} / 4$ & + & - & + & + & $\begin{array}{l}\text { DN hyperplasia } \\
\text { CC immaturity }\end{array}$ \\
\hline
\end{tabular}

$\mathrm{ARCN}=$ arcuate nucleus; $\mathrm{CC}=$ cerebellar cortex; $\mathrm{DN}=$ dentate nucleus; $\mathrm{pBN}=$ pre-Bötzinger nucleus.

Finally, we correlated all the findings with the mothers' smoking habit. A significant correlation was evident between maternal smoking, developmental alterations of the HGN, and sudden death. In fact, 6 of the 10 unexplained stillbirths and 12 of the 14 SIDS victims with HGN pathological findings, had smoker mothers $(p<0.05)$.

\section{Discussion}

Our interest in the HGN has been raised because of numerous experimental works showing the crucial role of this nucleus in the control of tongue movements particularly in inspiratory-related activity.

Despite these studies, only little research has been done, and only in the past, on the HGN in man. Precisely, alterations even discordant have been reported in SIDS victims. Naeye et al. [17] indicated a neuronal deficit in the HGN in more than two thirds of SIDS victims. O'Kusky and Norman $[18,19]$, through application of morphometric analysis, found increased volume associated to decreased neuronal density of this nucleus in SIDS cases than in controls. On the contrary, Konrat et al. [20] found a smaller average volume and increased density of neurons of the HGN in SIDS infants.

Therefore, our study was undertaken, besides to obtain basic information about the structure and physiology of the HGN during the first phases of human nervous system development, to make clear the morphological and biological features of the HGN not only in SIDS but even in victims of sudden unexplained perinatal death.

Through application of histological, morphometrical and immunohistochemical methods, we observed developmental anomalies of the HGN in high percentage of unexplained fetal and infant deaths, whereas such anomalies were not present in control cases.

Above all in SIDS group, a very high incidence of HGN pathological findings was found (14/15 cases). We identified four types of alteration: hyperplasia, 

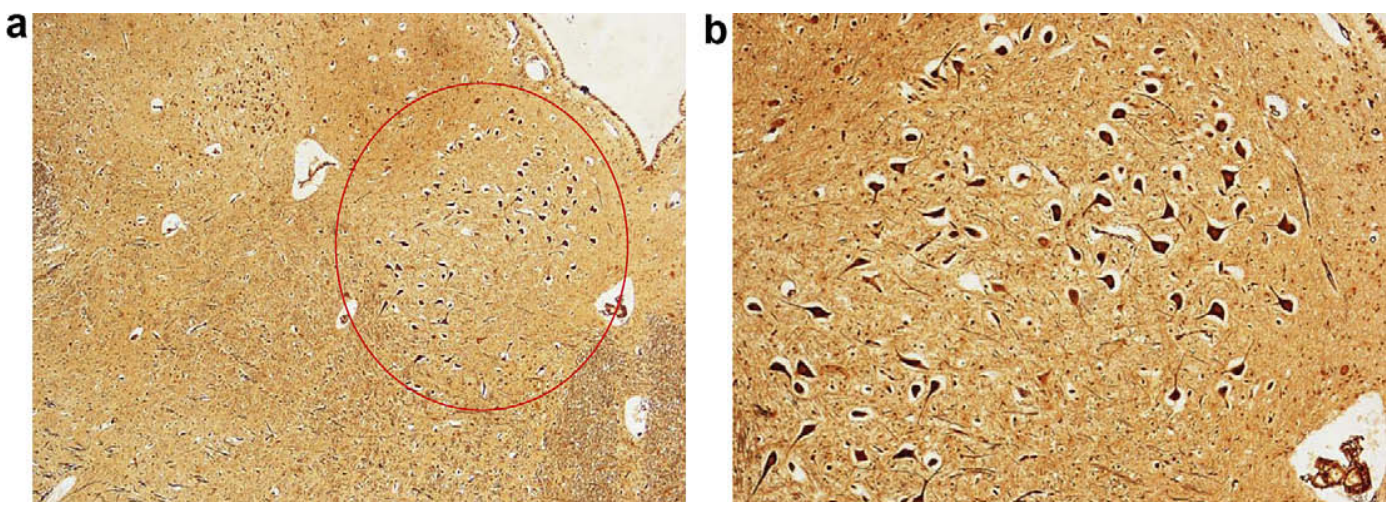

Fig. 6. Hyperplasia of the hypoglossal nucleus with increase in motoneuron number in a sudden infant death syndrome victim, 2 month-old. KlüverBarrera stain. Magnification: (a) $4 \times$; (b) $20 \times$.
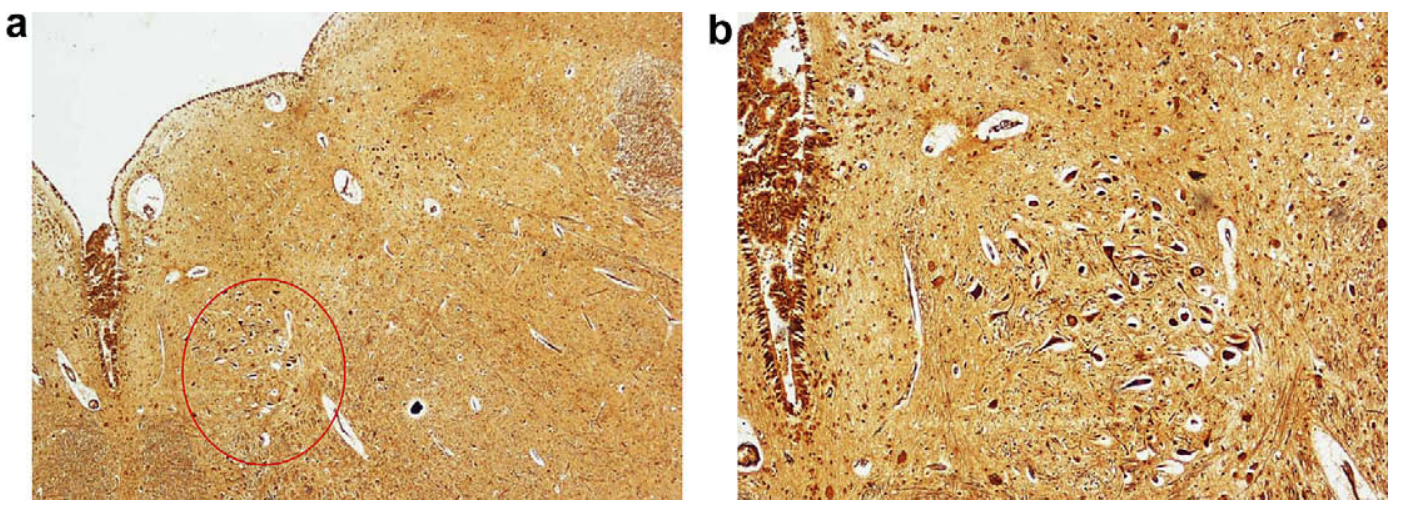

Fig. 7. Hypoplasia of the hypoglossal nucleus with decrease in motoneuron number in a sudden infant death syndrome victim, 2 month-old. KlüverBarrera stain. Magnification: (a) $4 \times$; (b) $20 \times$.

hypoplasia, positive expression of somatostatin, and absence of interneurons. The last anomaly was the most frequent finding, diagnosed in over half of SIDS victims.

The interneurons, that are small in size cells with thin axons and dendrites branching within the anatomic confines of the hypoglossal nucleus, are presumed to be inhibitory neurons because they use predominantly $\gamma$ aminobutyric acid (GABA) as a neurotransmitter [29]. Precisely, the interneurons are involved in the generation of inhibitory post-synaptic potentials projecting to the HGN motoneurons, that send their axons, by means of the hypoglossal nerve, to provide the motor innervation of the tongue [14-16].

In particular the genioglossus muscle of the tongue must contract rhythmically, by an excitation-suppression sequence, during respiration, chewing and shallowing, with different function-related frequencies $[15,16]$. The rhythmicity is the result of inhibitory cyclic patterns produced by interneurons, that directly depolarize the HGN motoneurons to generate discharge patterns.

A confirmation of the important role of the HGN interneurons in breathing control rises in our opinion from the study of Popratiloff et al. [30]. These authors, through stereotaxic microinjection of retrograde and anterograde neuronal tracers into the brainstem of rats, demonstrated that HGN interneurons project to the facial nucleus, thus sustaining the involvement of the HGN not only in tongue movements but also in orofacial coordination of the mimetic muscles.

Nevertheless, a consensus is now emerging in the literature that the function of hierarchical modulation of the respiratory rhythm is ascribed to the parafacial respiratory group, a pool of neurons defined as "pre-inspiratory neurons" surrounding and intermingled among the facial nucleus neurons. Besides, Zhang et al. [31] in experimental studies showed that even the facial nucleus takes part in the regulation of the respiratory activity. Thus, this strict relation with the facial/parafacial complex supports the leading role of the HGN interneurons in respiratory activity.

The absence of interneurons by us remarked in HGN of SIDS victims, can prevent the sequential rhythmic activity of the motoneurons and consequently the precise coordination of tongue movements, providing a potential anatomical substrate for respiratory and/or swallowing failure, possible causes of sudden death in the first months of life. 

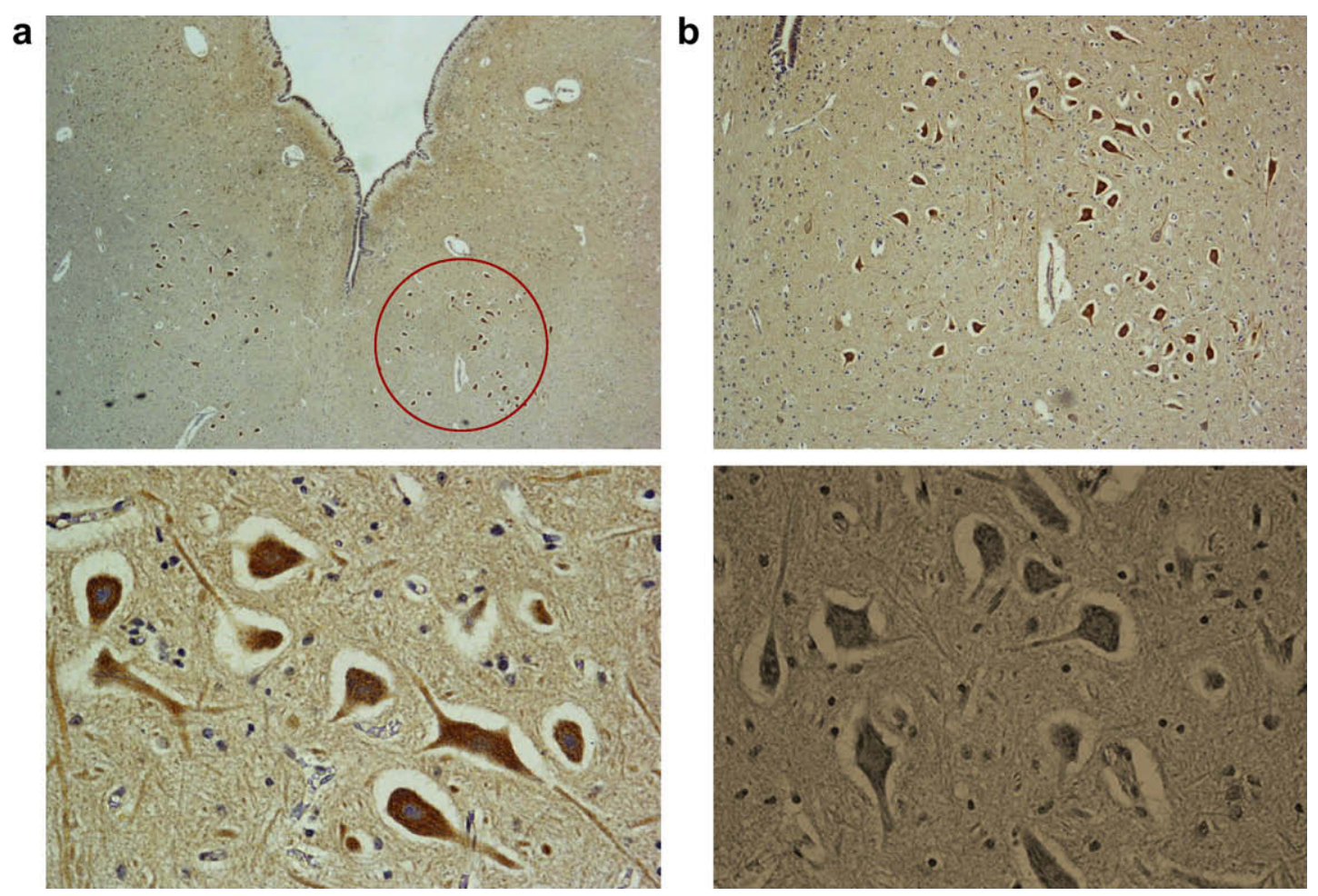

Fig. 8. Somatostatin-immunopositivity of the hypoglossal nucleus in a sudden infant death syndrome victim, 3 month-old. (a) immunopositive hypoglossal nucleus on both side of medulla oblongata (magnification $4 \times$ ); (b) monolateral immunoreactive neurons at greater magnification (10 $\times$ ); (c) single neurons. Note the positive staining in the neuronal cytoplasm $(40 \times)$; (d) an example of normal somatostatin-immunonegative HGN neurons in an age-matched infant of the control group (magnification $40 \times$ ).

The lack of interneurons, together with HGN hypoplasia and/or hyperplasia frequently remarked by us in SIDS group, are expression of common troubles arising during the central nervous system development.

Both hypoplasia and hyperplasia can be interpreted as results of a defect of the natural neuronal selection during the nervous system development [32]. This is a cell loss that occurs by a highly regulated process known as "programmed cell death" (PCD) for the reaching of the right size (number) of the precursor population of the neuronal structures and for the establishment and refinement of the related functional networks. The most generally accepted idea is in fact that neurons are produced in excess in order that they may compete for contacts with their cellular partners and thus adjust their numbers so as to provide sufficient innervation of their targets [33].

However, many of the neuronal populations in which developmental PCD occurs can make errors by reducing (giving rise to hyperplasia) or increasing (giving rise to hypoplasia) the selective removal of neurons in a determinate nucleus and/or structure. Both these defective processes can have the same repercussion and create similar disorders in the neuronal complex and its specific synaptic circuitry.

Another interesting result of this study is the different expression of the neurotransmitter somatostatin in the hypoglossus nucleus in cases of unexplained death com- pared to cases died of known etiology. We observed HGN-negativity for somatostatin in 6 of the 16 unexplained stillbirths and in no fetal death victim who died of known cause. On the contrary, among the infant deaths, the HGN was somatostatin-immunopositive in 6 of the 15 SIDS cases and immunonegative in all the controls.

Somatostatin is a neuropeptide with a wide distribution in the central nervous system, particularly in brainstem nuclei checking vital functions, during intrauterine life. After birth, the somatostatin concentration immediately decreases. Its presence is restricted to the perikarya of a small number of neurons.

Somatostatin seems to exert important inhibitory action on respiration. In support of this hypothesis, it has been shown that intracisternal administration or microinjections of this neurotransmitter in the ventral medulla of rats produces deep and prolonged apnea [34-36]. In addition, intravenous injection of somatostatin in human depresses the ventilatory response to hypoxia [37,38].

Therefore, we assume that the high diffusion of the somatostatin in brainstem respiratory centers in fetal life is strongly involved in the inhibitory action on the intrauterine breathing and that the low concentration of this neurotransmitter after birth is essential to allow the regular ventilatory rhythm. 
The alterations of the somatostatin-expression that we observed in this study in both sudden fetal and infant deaths, and precisely the presence of HGN somatostatin-immunonegative and positive neurons before and after birth, respectively, even more if they are associated to HGN morphological alterations, may have deleterious effects on the respiratory activity, leading to fatal breathing movements in fetuses and to drawback of the normal sequence of genioglossus muscle contraction during inspiration after birth, up to irreversible apnea.

Thus, we detain that the normal structural and functional development of the HGN is vital for the generation of the respiratory network and any disturbance in its maturative process may be lethal already in perinatal life.

In addition to the above-described HGN findings, our results indicate a significant association between these lesions and developmental alterations of different brainstem and cerebellum structures.

We detain that the susceptibility to SIDS and SIUD occurs through a mechanism involving morphological developmental defects of various centers that, being linked one with the other through specific synaptic networks, are together important in the modulation of all the vital functions. Consequently, the developmental alterations of these neuronal structures can concur to produce severe dysregulation of the autonomic nervous system homeostatic control, triggering the death mechanism.

The abnormalities of the HGN, such as of the other structures observed in this study, may be the result of the negative effect of environmental factors, and above all of maternal smoking in pregnancy, on the developing brain.

At present, there is a general consensus that cigarette smoking during pregnancy is noxious already to the fetus, and results in deficits such as retarded intrauterine growth, low birth weight and stillbirth [39-41]. Besides, several studies have shown that nicotine interferes even with the normal course of the brain development $[25,42,43]$. Previously, we have reported that prenatal nicotinic exposure affects the development of nervous system centers checking vital functions [21,24-26].

In the present study we found an important association even between prenatal cigarette exposure, functional and/or structural alterations of the HGN and sudden fetal/infant death. In fact, 18 among the 24 victims of unexplained death with HGN wrong maturation (75\%), had smoker mothers.

Therefore, on the basis of the results of this study and of our previous researches, we hypothesize that exposure in utero to maternal smoking could interfere with brain development, giving rise to a widespread incorrect maturation of all the neuronal structures involved in control of the vital functions.

The role of different risk factors, besides cigarette smoking, as possible cause for fetal and infant death must also be stressed. For example, as most of the moth- ers and infants of the study lived in large cities, we assume, in agreement with a recent study linking air pollutants to adverse birth outcomes [44], that atmospheric pollution may have contributed to the development of the above reported lesions.

In conclusion, given the impossibility to avoid air pollution, we anyway recommend clinical and public health strategies aimed at the prevention of prenatal tobacco exposure of infants. Primary care should inform young women about the long-term consequences of smoking during pregnancy.

\section{References}

[1] Matturri L, Minoli I, Lavezzi AM, Cappellini A, Ramos S, Rossi L. Hypoplasia of medullary arcuate nucleus in unexpected late fetal death (stillborn infants): a pathologic study. Pediatrics 2002;109:E43.

[2] Matturri L, Ottaviani G, Alfonsi G, Crippa M, Rossi L, Lavezzi AM. Study of the brainstem, particularly the arcuate nucleus, in sudden infant death syndrome (SIDS) and sudden intrauterine unexplained death (SIUD). Am J Forensic Med Pathol 2004;25:44-8.

[3] Lavezzi AM, Ottaviani G, Mauri M, Matturri L. Hypoplasia of the arcuate nucleus and maternal smoking during pregnancy in sudden unexplained perinatal and infant death. Neuropathology 2004;24:284-9.

[4] Lavezzi AM, Matturri L. Functional neuroanatomy of the human pre-Bötzinger complex with particular reference to sudden unexplained perinatal and infant death. Neuropathology 2008;28:10-6.

[5] Biondo B, Magagnin S, Bruni B, Matturri L. Glial and neuronal alterations in the nucleus tractus solitarii of sudden infant death victims. Acta Neuropathol (Berl) 2004;108:309-18.

[6] Lavezzi AM, Matturri L. Hypoplasia of the parafacial/facial complex: a very frequent finding in sudden unexplained fetal death. Open Neurosci J 2008;2:1-5.

[7] Lavezzi AM, Ottaviani G, Ballabio G, Rossi L, Matturri L. Preliminary study on the cytoarchitecture of the human parabrachial/Kölliker-Fuse complex with reference to sudden infant death syndrome and sudden intrauterine unexplained death. Pediatr Dev Pathol 2004;7:171-9.

[8] Roda F, Gestreau C, Bianchi AL. Discharge patterns of hypoglossal motoneurons durino fictive breathing, coughing and swallowing. J Neurophysiol 2002;87:1703-11.

[9] Withington-Wray DJ, Mifflin SW, Spyer KM. Intracellular analysis of respiratory-modulated hypoglossal motoneurons in the cat. Neuroscience 1988;25:1041-51.

[10] Ono T, Ishiwata Y, Kuroda T, Nakamura Y. Swallowing-related perihypoglossal neurons projecting to hypoglossal motoneurons in the cat. J Dent Res 1998;77:351-60.

[11] Cooper MH. The hypoglossal nucleus of the primate: a Golgi study. Neurosci Lett 1981;21:249-54.

[12] Boone T, Aldes LD. The ultrastructure of two distinct neuron populations in the hypoglossal nucleus of the rat. Exp Brain Res 1984;54:321-6.

[13] Jankowska E, Roberts WJ. Synaptic actions of single interneurons mediating reciprocal inhibition of motoneurones. J Physiol 1972;222:623-42.

[14] Withington-Wray DJ, Mifflin SW, Spyer KM. Intracellular analysis of respiratory-modulated hypoglossal motoneurons in the cat. Neuroscience 1988;25:1041-51.

[15] Lowe AA. Excitatory and inhibitory input to hypoglossal motoneurones and adjacent reticular formation neurones in cat. Exp Neurol 1978;62:30-47. 
[16] Lowe AA. The neural regulation of tongue movements. Prog Neurobiol 1980;15:295-344.

[17] Naeye RL, Olsson JM, Combs JW. New brainstem and bone marrow abnormalitiesin victims of sudden infant death syndrome. J Perinatol 1989;9:180-3.

[18] O'Kusky JR, Norman MG. Sudden infant death syndrome: postnatal changes in the numerical density and total number of neurons in the hypoglossal nucleus. J Neuropathol Exp Neurol 1992;51:577-84.

[19] O'Kusky JR, Norman MG. Sudden infant death syndrome: increased number of synapses in the hypoglossal nucleus. $\mathbf{J}$ Neuropathol Exp Neurol 1995;54:627-34.

[20] Konrat G, Halliday G, Sullivan C, Harper C. Preliminary evidence suggesting delayed development in the hypoglossal and vagal nuclei of SIDS infants: a necropsy study. J Child Neurol 1992;7:44-9.

[21] Lavezzi AM, Ottaviani G, Mauri M, Matturri L. Alterations of biological features of the cerebellum in sudden perinatal and infant death. Curr Mol Med 2006;6:429-35.

[22] Lavezzi AM, Ottaviani G, Terni L, Matturri L. Histological and biological developmental characterization of the human cerebellar cortex. Int J Dev Neurosci 2006;24:365-71.

[23] Lavezzi AM, Ottaviani G, Matturri L. Ontogenesis of human cerebellar cortex and biopathological characterization in sudden unexplained fetal and infant death. Virchows Arch 2007;450:31-40.

[24] Lavezzi AM, Ottaviani G, Mingrone R, Matturri L. Analysis of the human locus coeruleus in perinatal and infant sudden unexplained death. Possible role of the cigarette smoking in the development of this nucleus. Brain Res Dev Brain Res 2005;154:71-80.

[25] Lavezzi AM, Ottaviani G, Matturri L. Adverse effects of prenatal tobacco smoke exposure on biological parameters of the developing brainstem. Neurobiol Dis 2005;20:601-7.

[26] Lavezzi AM, Ottaviani G, Mauri M, Matturri L. Biopathology of the olivocerebellar network in sudden unexplained perinatal and sudden infant death syndrome related to maternal cigarette smoking. Neurol Res 2007;29:525-32.

[27] Matturri L, Ottaviani G, Lavezzi AM. Techniques and criteria in pathologic and forensic-medical diagnostics of sudden unexpected infant and perinatal death. Am J Clin Pathol 2005;124:259-68.

[28] Matturri L, Ottaviani G, Lavezzi AM. Guidelines for neuropathologic diagnostics of perinatal unexpected loss and sudden infant death sindrome (SIDS). A technical protocol. Virchows Arch 2008;452:19-25.

[29] Takasu N, Nakatani T, Arikuni T, Kimura H. Immunocytochemical localization of gamma-aminobutyric acid in the hypoglossal nucleus of the Macaque monkey. A light and electron microscopic study. J Comp Neurol 1987;263:42-53.

[30] Popratiloff AS, Streppel M, Gruart A, Guntinas-Lichius O, Angelov DN, Stennert E, et al. Hypoglossal and reticular interneurons involved in oro-facial coordination in the rat. $\mathrm{J}$ Comp Neurol 2001;433:364-79.

[31] Zhang C, Yan H, Li C, Zheng Y. Possible involvement of the facial nucleus in regulation of respiration in rats. Neurosci Lett 2004;367:283-8.

[32] Buss RR, Sun W, Oppenheim RW. Adaptive roles of programmed cell death during nervous system development. Annu Rev Neurosci 2006;29:1-35.

[33] Pettmann B, Henderson CE. Neuronal cell death. Neuron 1998;20:633-47.

[34] Chen Z, Hedner T, Hedner J. Local application of somatostatin in the ventrolateral brain medulla induces apnea in the rat. J Appl Physiol 1990;69:2233-8.

[35] Harfstrand A, Kalia M, Fuxe K, Kaijser L, Agnati LF. Somatostatin induced apnoea: interaction with hypoxia and hypercapnea in the rat. Neurosci Lett 1984;50:37-42.

[36] Kalia M, Fuxe K, Agnati LF, Hökfelt T, Härfstrand A Somatostatin produces apnoea and is localized in medullary respiratory nucleus: a possible role in apnoic syndromes. Brain Res 1984;296:339-44.

[37] Filuk RB, Berezanski DJ, Anthonisen NR. Depression of hypoxic ventilatory response in humans by somatostatin. J Appl Physiol 1988;65:1050-4.

[38] Maxwell DL, Chahal P, Nolop KB, Hughes JM. Somatostatin inhibits the ventilatory response to hypoxia in humans. J Appl Physiol 1986;60:997-1002.

[39] Cnattingius S, Axelsson O, Eklund G, Lindmark G. Smoking, maternal age and fetal growth. Obstet Gynecol 1985;66:449-52.

[40] Cole PV, Hawkins LH, Roberts D. Smoking during pregnancy and its effects on the fetus. J Obstet Gynaecol Br Commonw 1972;79:782-7.

[41] Kalousek DK, Gilbert-Barness E. Causes of stillbirth and neonatal death. In: Gilbert-Barness E, editor. Potter's Pathology of the Fetus and Infant. St. Louis, MO: Mosby; 1997. p. 128-62.

[42] Gospe SM, Zhou SS, Pinkerton KE. Effects of environmental tobacco smoke exposure in utero on brain development. Pediatr Res 1996;39:494-8.

[43] Lichtensteiger W, Ribary U, Schlumpf M, Odermatt B, Widemer HR. Prenatal adverse effects of nicotine on the developing brain. Prog Brain Res 1988;73:137-57.

[44] Ritz B, Wilhelm M. Ambient air pollution and adverse birth outcomes: methodologic issues in an emerging field. Basic Clin Pharmacol Toxicol 2008;102:182-90. 